\title{
Grundversorgernetzwerke statt ANOVA?
}

Das Informationsschreiben eines Grundversorgernetzwerkes lässt aufhorchen: Es hat sich mit den Versicherern auf «definierte Managed-Care-Regeln» geeinigt. Spezialisten werden darin verpflichtet, beim Grundversorger Rücksprache zu nehmen, wenn Abklärungen und Behandlungen ausserhalb des expliziten Überweisungsauftrages durchgeführt werden. Wohin führt das?

Jürg Nadig

Präsident der Schweizerischen Gesellschaft für Medizinische Onkologie SGMO und des Dachverbandes der Swiss Federation of Specialities in Medicine SFSM
Korrespondenz:

Dr. med. Jürg Nadig MAE Facharzt für Medizinische Onkologie und Innere Medizin Bannhaldenstrasse 7

CH-8180 Bülach

Tel. 0448627300

Fax 0448627301

juerg.nadig@hin.ch
Neue Bundesgerichtsentscheide zu den Wirtschaftlichkeitsverfahren stärken die Position der Ärzte. Um mit der Statistischen Methode die Wirtschaftlichkeit zu prüfen, muss der Beklagte mit einem vergleichbaren Kollektiv von mindestens 10 Ärzten über eine genügend lange Periode verglichen werden [1]. Damit anerkennt das Bundesgericht die Forderung der Schweizerischen Gesellschaft für Medizinische Onkologie SGMO, dass nur Gleiches mit Gleichem verglichen werden kann. Es verpflichtet die Versicherer, die Vergleichbarkeit des Kollektivs nachzuweisen, und gibt den medizinischen Onkologen recht, die zeigen konnten, dass in ihrem Fach die Fallkosten abhängig sind vom Anteil Patienten, die eine Chemotherapie erhalten, vom Casemix und von der Therapiestrategie [2]. Letztere hat zudem einen Einfluss auf das Überleben. Neben der neuen Rechtsprechung werden parlamentarische Vorstösse zu einer verbesserten Wirtschaftlichkeitsprüfung führen, und die geringer werdende Abdeckung des Datenpools der Santésuisse schwächt die Position der Versicherer in WZW-Verfahren (Wirksamkeit, Zweckmässigkeit, Wirtschaftlichkeit) weiter. Was läge also näher, als neue Sanktionsmöglichkeiten für «unwirtschaftliche» Spezialisten zu suchen? gen in Zukunft konkret aus? Hat er bei Husten im Rahmen einer bleomycinhaltigen Therapie zuerst den Hausarzt anzufragen, ob er eine CO-Diffusion oder eine Dünnschicht-CT zum Ausschluss einer beginnenden Bleomycinlunge veranlassen darf? Muss er zuerst die Hausärztin fragen, ob Blutkulturen und Urikult sowie ein Thorax-Rx angefertigt werden dürfen, wenn ein Patient im Rahmen einer Chemotherapie eine febrile Neutropenie entwickelt und innert drei Stunden eine antibiotische Therapie begonnen werden muss? Wie formuliert der Grundversorger den expliziten Behandlungsauftrag beim Rektumkarzinom, bei dem mindestens fünf Spezialisten involviert sind, und wer koordiniert Abklärung und Behandlung? Leider fehlen die Grundversorger aus Zeitmangel in der Regel an den entsprechenden interdisziplinären Tumorboards.

Mit den vereinbarten Managed-Care-Regeln werden «die Grundversorger zur Kostenkontrolle verpflichtet. Wenn sie in den Rechnungskontrollen Abklärungen erkennen, die nicht abgesprochen wurden und die sie [die Grundversorger] als unnötig erachten, können sie die Vergütung der verursachten Leistung durch die Krankenkasse ablehnen. (...) Nach Ablauf einer gewissen Vertragsdauer (1-3 Jahre) sind

\section{Muss der Spezialist bei Husten im Rahmen einer bleomycinhaltigen Therapie zuerst den Hausarzt anfragen, ob er eine CO-Diffusion oder eine Dünnschicht-CT veranlassen darf?}

Ein Informationsschreiben eines Grundversorgernetzwerkes lässt aufhorchen. Das Netzwerk hat sich kürzlich mit den Versicherern auf «definierte Managed-Care-Regeln geeinigt». Mit dem Vertrag, den lediglich die Grundversorger abgeschlossen haben, verpflichten sie nun die Spezialisten, die ja nicht Teil der Vereinbarung sind, «beim Grundversorger Rücksprache zu nehmen, wenn Abklärungen und Behandlungen ausserhalb des expliziten Überweisungsauftrages durchgeführt werden». Wie sieht das für den Onkolo- wir verpflichtet, uns um Einsparungen zu bemühen, das heisst, (...) mit sparsamen Spezialisten zusammenzuarbeiten.»

Wie können Netzwerke das schaffen, was santésuisse bei Wirtschaftlichkeitsverfahren bis jetzt nicht schaffte: Homogene Vergleichskollektive von Spezialisten zu finden? Wie kann da ein einzelner Grundversorger oder ein Netzwerk mit ein paar Dutzend Grundversorgern entscheiden, ob ein Onkologe eine billigere, aber suboptimale Medizin betreibt oder eine 


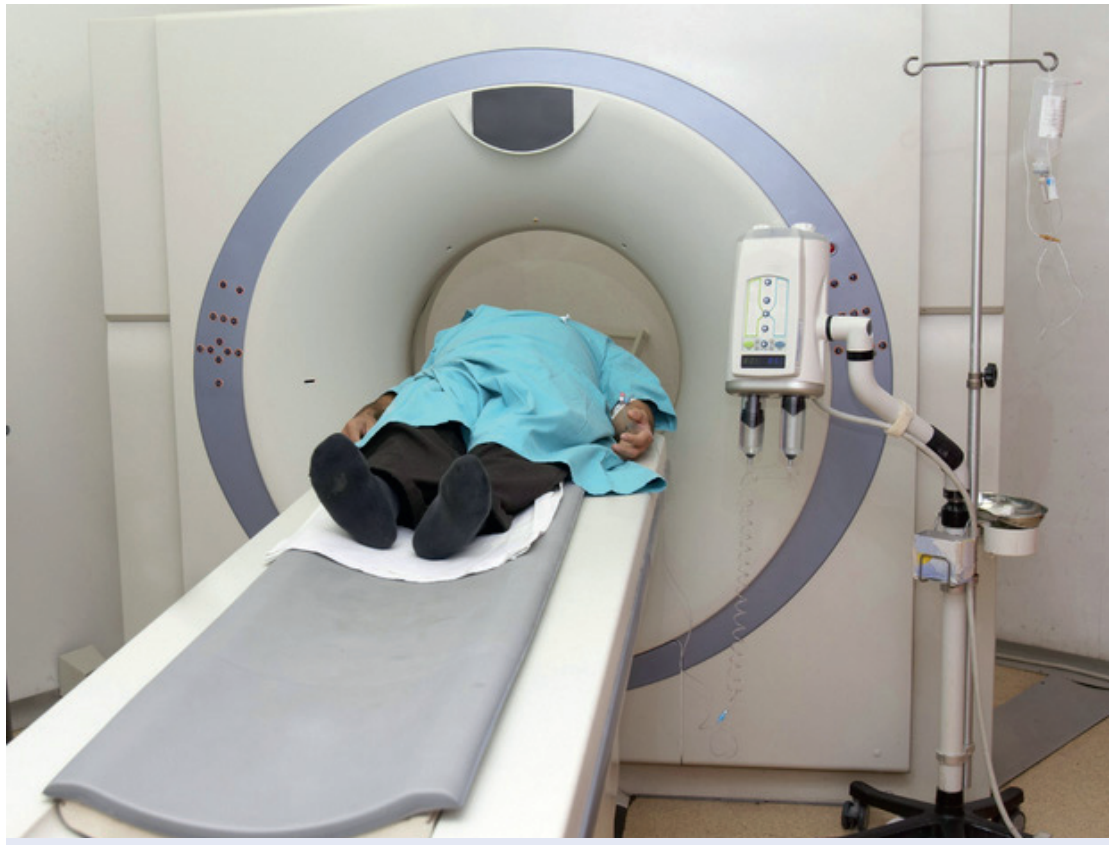

Muss der Spezialist demnächst erst den Hausarzt fragen, wenn er weiterführende Untersuchungen ausserhalb des expliziten Überweisungsauftrages durchführen will? kostenintensivere aber optimale? Versuchen die Versicherer, die neuen höchstrichterlichen Auflagen zu den Wirtschaftlichkeitsverfahren zu umgehen, indem sie den Grundversorgern Aufgaben überbürden, zu denen ihnen zumindest die statistischen Daten fehlen, auch wenn sie die fachliche Kompetenz noch besässen?
Unschuld waschen? Delegieren die Versicherer die Rechnungskontrollen an die Netzwerke, weil ihnen das Fachwissen zur Beurteilung fehlt? Oder haben die Versicherer eingesehen, dass diese Form von Kontrolle ein zu grosses einklagbares Willkürpotential in sich birgt und wenig hilfreich ist, Strukturen $\mathrm{zu}$ optimieren?

Managed-Care-Projekte haben dann für den Patienten einen Nutzen, wenn integrierte Versorgungsstrukturen mit partnerschaftlichem Einbezug der Spezialisten entstehen. In der Onkologie sind zentrale Strukturelemente gemeinsam ausgearbeitete Behandlungspfade, die Offenlegung von Behandlungsdaten in Registern und die Vollkostenerfassung für die ganze Behandlungskette [3].

Brent James Warnung, Managed Care meine nicht Steuerung der Ärzte und Pflegenden durch Administratoren, sondern Steuerung der Behandlungsprozesse durch Kliniker im Interesse des Patienten, gilt auch für Grundversorgernetzwerke, welche die Rolle der Administratoren übernehmen wollen [4].

Netzwerkvertreter und Parlamentarier beteuern immer wieder, Managed-Care-Verträge seien keine Sparmodelle, sondern dienten der Qualitätsverbesserung. santésuisse versichert, die heute etablierten Behandlungen in der Onkologie müssten allen Patienten zugute kommen, unabhängig davon, ob sie in einem Managed-Care-System oder in einem Modell mit freier Arztwahl versichert seien. Wenn die Information dieses Netzwerks die zukünftige Wirklichkeit widerspiegelt, kommen Zweifel auf ob der hehren Worte der Politik. Vielmehr wäre zu fürchten, dass

\section{Vielmehr ist zu fürchten, dass solche Netzwerke Treiber einer impliziten Rationierung werden. Da wäre sogar ANOVA falschen Freunden vorzuziehen.}

Waren WZW-Verfahren unangenehm, so konnte sich der beklagte Arzt zumindest vor einem unabhängigen Gericht rechtfertigen. Dieses Netzwerk arbeitet aber einfach nicht mehr mit dem scheinbar teuren Arzt zusammen, ohne dass er sich vor einem unabhängigen Dritten zur Wehr setzen kann. Der Spezialist ist in diesem Netzwerk nicht mehr der santésuisse mit ihren ruinösen Rückforderungen ausgeliefert, sondern seine Existenz ist vom Wohlwollen eines Netzwerkes abhängig, das über noch weniger Vergleichszahlen verfügt als der Datenpool der santésuisse. Gibt es eine paritätische Schlichtungskommission, die solche beanstandeten Rechnungen prüft und als unabhängiges Gremium entscheiden kann? Was ist der hier vorgesehene Rechtsweg? Welche Vorkehrungen gibt es, dass der Grundversorger nicht in eigener Sache richtet, während die Versicherer die Hände in solche Netzwerke Treiber einer impliziten Rationierung würden. Da wäre sogar ANOVA falschen Freunden vorzuziehen. Cavete, collegae!

\section{Literatur}

1 Referenz 9C_968/2009, http://jumpcgi.bger.ch/ cgibin/JumpCG?id=15.12.2010_9C968/2009

2 Nadig J. Verdeckte Rationierung dank Wirtschaftlichkeitsverfahren? Schweiz Ärztezeitung. 2008;89(20):855-60.

3 Steuerung komplexer Systeme: Alles aus zwei Händen: Spezialist und Grundversorger. Gemeinsames Symposium von Spezialisten und Grundversorgern. J. Fritschi, P. Berchtold, J. Nadig an der 78. Jahrestagung der SGIM 2010 in Basel.

4 An Introduction to Clinical Quality Improvement. http://intermountainhealthcare.org/xp/public/ documents/institute/intro.ppt 